\title{
SI TEXT Recovering Protein Thermal Stability Using All-Atom Hamiltonian Replica-Exchange Simulations in Explicit Solvent
}

\author{
Guillaume Stirnemann* and Fabio Sterpone ${ }^{\dagger}$ \\ CNRS Laboratoire de Biochimie Théorique, Institut de Biologie Physico-Chimique, \\ Univ. Paris Denis Diderot, Sorbonne Paris Cité, PSL Research University, \\ 13 rue Pierre et Marie Curie, 75005, Paris, France
}

(Dated: November 12, 2015)

\section{REST2 IMPLEMENTATION}

We have modified and adapted the original temperature-exchange TcL routine of NAMD and we have created new TcL functions able to rescale the force-field parameters of a given set of atoms. Following ref, ${ }^{1}$ the exchange acceptance ratio has been modified to satisfy the detailed balance conditions in the NVT ensemble. In the NAMD formalism, replicas that run on distinct physical cores periodically exchange the scaling factors $\lambda_{i}$. Our modified version of the code and the TcL routines have been compiled and successfully ported on many different platforms, including BlueGene/Q.

\section{SIMULATION DETAILS}

The crystal and NMR structures of CLN025 (YYDPETGTWY) have been reported ${ }^{2}$ but were not deposited in the Protein DataBank at the moment of the system preparation (they were deposited after this work was completed (NMR (2RVD) and crystal (5AWL)). We mutated the $\mathrm{N}$ - and C-terminal of PDB crystal structure of the original chignolin design $(1 \mathrm{UAO})^{3}$ whose native structure was shown to be very close to that of CLN025. ${ }^{2}$ This protein was modeled using the CHARMM36 forcefield ${ }^{4}$ and solvated in a box of 1,811 TIP3P water molecules and 2 sodium ions. It was minimized using the steepest-descent algorithm as implemented in the software NAMD $2.9^{5}$ and equilibrated with the same program in the NPT ensemble $(P=1$ bar, $T=300 \mathrm{~K})$ for $2 \mathrm{~ns}$. The final structure was taken as the the initial state for all replicas of the subsequent REST2 study. For all the simulations, we used a PME grid size of $1.2 \AA$, a timestep of $2 \mathrm{fs}$, and a vdW interaction cutoff of $10 \AA$.

Starting from this previously equilibrated, folded protein, configurations of CLN025 in explicit solvent, we have performed REST2 simulations in the NVT ensemble at a reference physical temperature of $\beta_{\text {ref }}=300 \mathrm{~K}$ for 300 ns on 12 replicas exchanging the following proteinprotein corresponding temperatures: 290, 300, 311, 322, 333, 345, 368, 394, 423, 455, 491 and $529 \mathrm{~K}$. Exchanges were attempted every $10 \mathrm{ps}$. These temperatures were chosen so that the overlap between the relevant energy contributions and the exchange probability were satisfying.

A similar methodology was employed to simulate the original design of chignolin CLN001 (GYDPETGTWG) starting from its original $1 \mathrm{UAO}$ crystal structure. ${ }^{3}$ As this protein is only marginally stable at room temperature, we had to chose a temperature range different from that described above to ensure appropriate sampling of the protein folded state. We used the following protein-protein temperature-range, with approximately half of the replicas below the experimental melting temperature, and half above: $252.2,260.9,270.5,280,289.6$, 300, 320.0, 342.6, 367.8, 395.7, 427.0, 460.1 K. All the rest was identical to the simulations of CLN025.

REMD simulations were performed starting from same initial configurations of CLN025 and were propagated using a methodology identical to that used earlier for CLN001, ${ }^{6}$ and with the same temperature distributions (32 replicas spanning a temperature range between $278 \mathrm{~K}$ and $600 \mathrm{~K})$.

Finally, our original REST2 simulations were further propagated at two higher reference temperatures $(333 \mathrm{~K}$ and $368 \mathrm{~K}$ ), keeping the same temperature distributions (this required to change the $\lambda$-distribution accordingly). Since the solvent density is temperaturedependent, maintaining the original $300-\mathrm{K}$ volume would lead to average pressures larger than 1 bar, which may affect protein stability. This problem was avoided by performing these simulations in the isobaric-isothermic ensemble. Since the pressure and the temperature are similar among replicas, no change is required in the detailed balance condition. ${ }^{7,8}$ Simulations were propagated for $100 \mathrm{~ns}$, where the first 50-ns were used for equilibration and the final $50 \mathrm{~ns}$ for production.

\section{COLLECTIVE VARIABLES}

The root-mean-square displacement (RMSD) used in the main text was defined with respect to the center of the most populated cluster in the native state. We employed a clustering analysis of a 50-ns portion of the trajectory with $\lambda=1$ using a cutoff of $2 \AA$. Then, the folded state confirmations used for the estimation of the protein stability curves were those with $\mathrm{RMSD}<2 \AA$. For chignolin, the precise value of this cutoff has very little impact on the validity of the results as the region between 1 and $3 \AA$ is very sparsely populated in the simulation (see e.g. Fig. 1D). Any dividing surface between these values would therefore lead to the same results.

As an alternative, we also computed the RMSD with 
respect to the crystal structure of the original chignolin design 1UAO, which was the only one deposited in the Protein DataBank. While the RMSD in the folded state was on average slighty higher than the one following the previous definition $(1.2 \AA$ vs $0.8 \AA)$, the fraction of folded states defined this way was undistinguishable from that following the definition based on the center of the most populated cluster. The comparison to the original chignolin structure is motivated by several observations. First, it was reported that the tertiary structure of CLN025 was actually very close to that of the original chignolin, ${ }^{2}$ showing especially the same key contacts in the hairpin structure. Second, the RMSD between the NMR and crystal structures of CLN025 is equal to $1.75 \AA^{2}$ and is actually larger than the RMSD between the center of the most populated cluster of CLN025 in the simulations at ambient temperature and the crystal structure of chignolin $1 \mathrm{UAO}$, which is equal to $1.27 \AA$.

Finally, we have compared the results obtained using the RMSD as a collective variable to that using a reaction coordinate based on the number of contacts. We used here the methodology detailed in the SI of ref $^{9}$ to define a smoothed-version of the number of contacts $Q$ based on the most populated cluster defined above and the contacts present in the protein native state. A cutoff of 0.8 ( 1 being native, 0 being unfolded with no native contact) was employed. Once again, for such a small protein with a simple topology, the definition of the cutoff has only little influence on the final results.

\section{COMPARING THE STABILITY OF TWO MUTANTS}

In Figure 1, we compare the melting curves of CLN001 and of CLN025 on a $\lambda$-scale (last 150 ns of each 300-ns run). As evidenced by the experimental melting temperature shift $(\approx 30 \mathrm{~K})$, our simulations similarly reproduce this dramatic shift in the protein thermal stability upon two single-site mutations.

\section{JUSTIFICATION OF EQ. (3) IN THE MANUSCRIPT}

For a protein-water system evolving at the reference temperature $\beta_{\text {ref }}$ and controlled by the rescaled potential energy $E_{i}$ we can exploit the corresponding state principle to define the instantaneous, effective temperature $\beta_{i}^{\prime}$ associated to the potential energy rescaling. In the context of REST2, in each frame of each $i$ window this implies:

$$
\beta_{\text {ref }} E_{i}(\vec{X})=\beta_{i}^{\prime} E_{\text {ref }}(\vec{X})
$$

where $\vec{X}$ represents protein and solvent coordinates. Because of the asymmetric scaling of the potential energy terms encoded in the REST2 procedure we are tempted

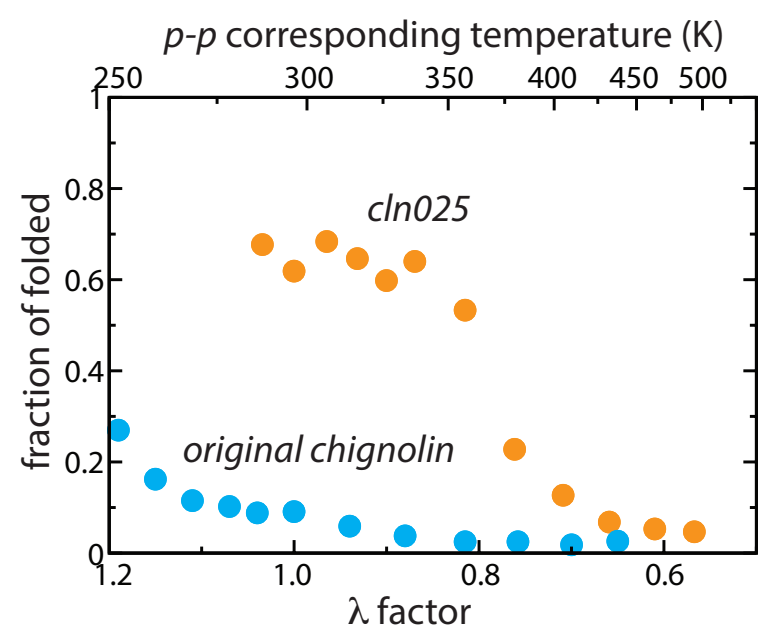

FIG. 1. REST2 simulations of the original CLN001 chignolin (blue circles) and of CLN025 (orange circles), last $150 \mathrm{~ns}$, using an RMSD-based cutoff to define the fraction of folded states. The temperature distribution was different for each peptide as the original chignolin design is only marginally stable at ambient temperature.

to focus only on the degrees of freedom of the protein and therefore deduce an effective temperature for the protein conformational equilibrium only. Making the approximation that we can treat the solvent contribution as a meanfield for the protein conformational equilibrium, we can write the corresponding state principle for the protein contribution including the mean effect of the solvent on the protein/solvent interaction (formally $\bar{E}_{p w}\left(\vec{X}_{p}\right)$ ) and neglecting the $E_{w w}$ which is unperturbed and which only depends upon the solvent atomic coordinates $\vec{X}_{w}$. Using the REST2 expression for protein-protein and proteinsolvent scaling we can write down an approximated version of Eq. 2:

$\beta_{r e f}\left(\lambda_{i} E_{p p}\left(\vec{X}_{p}\right)+\sqrt{\lambda_{i}} \bar{E}_{p w}\left(\vec{X}_{p}\right)\right)=\beta_{i}^{\prime}\left(E_{p p}\left(\vec{X}_{p}\right)+\bar{E}_{p w}\left(\vec{X}_{p}\right)\right)$

where $\vec{X}_{p}$ refers to the protein coordinates. Here above we have assumed that it is possible to average the proteinwater interactions over the solvent coordinates $\vec{X}_{w}$. By replacing $\lambda_{i}$ using $\beta_{i}=\lambda_{i} \beta_{\text {ref }}$, and rewriting the corresponding equation in a more compact form, we finally obtain:

$$
\beta_{i}^{\prime}=\beta_{i}\left(1+\left(\sqrt{\frac{\beta_{r e f}}{\beta_{i}}}-1\right) \frac{\bar{E}_{p w}\left(\vec{X}_{p}\right)}{E_{p p}\left(\vec{X}_{p}\right)+\bar{E}_{p w}\left(\vec{X}_{p}\right)}\right)
$$

This is the instantaneous protein effective temperature for each frame of each window $i$; we can finally average over all the frames of a given window to obtain the average of this effective temperature:

$$
\left\langle\beta_{i}^{\prime}\right\rangle=\beta_{i}\left(1+\left(\sqrt{\frac{\beta_{r e f}}{\beta_{i}}}-1\right)\left\langle\frac{\bar{E}_{p w}\left(\vec{X}_{p}\right)}{E_{p p}\left(\vec{X}_{p}\right)+\bar{E}_{p w}\left(\vec{X}_{p}\right)}\right\rangle\right)
$$


In practice, it is rather complicate to evaluate $\bar{E}_{p w}\left(\vec{X}_{p}\right)$ for each $\vec{X}_{p}$ directly. One strategy would consist in coarse-graining the protein configuration space via e.g. cluster analysis or projection onto a very good reaction coordinate. For each representative state of the protein conformation, independent simulations should be performed constraining the protein degree of freedom, and allowing solvent dynamics. However, it is actually much more simple to do the average at once in Eq. 4, i.e., to consider that the instantaneous value of protein/solvent interactions $E_{p w}\left(\vec{X}_{p}, \vec{X}_{w}\right)$ is close to its average over $\vec{X}_{w}$ positions, which is $\bar{E}_{p w}\left(\vec{X}_{p}\right)$. This is probably legitimate since the solvent molecule would adapt fast to any change in the slower $\vec{X}_{p}$ coordinates to minimize the energy, so that $\bar{E}_{p w}$ will be mostly governed by the protein configurations. The resulting equation used to evaluate the protein effective temperature is thus

$$
\left\langle\beta_{i}^{\prime}\right\rangle=\beta_{i}\left(1+\left(\sqrt{\frac{\beta_{r e f}}{\beta_{i}}}-1\right)\left\langle\frac{E_{p w}(\vec{X})}{E_{p p}\left(\vec{X}_{p}\right)+E_{p w}(\vec{X})}\right\rangle\right)
$$

While this equation is obtained after some approximations, the resulting temperature range and stability curve are in very good agreement with experimental data.

\begin{tabular}{|c|c|c|c|}
\hline Method & PDB & Mutation & $\Delta \Delta G_{u / f}[\mathrm{kcal} / \mathrm{mol}]$ \\
\hline PoPServer & 1UAO & G1Y/G10Y & 1.4 \\
Maestro & 1UAO & G1Y/G10Y & 0.7 \\
Eris rbb & 1UAO & G1Y/G10Y & -3.0 \\
Eris fbb & 1UAO & G1Y/G10Y & -4.0 \\
\hline Eris rbb & 2RVD & Y1G/Y10G & 2.6 \\
Eris fbb & 2RVD & Y1G/Y10G & 1.2 \\
\hline FEP & 1UAO & G1Y/G10Y & -1.0 \\
\hline
\end{tabular}

TABLE I. Results of single-point mutation calculations. For each method, the resulting change in the free-energy difference between the folded and unfolded configurations is given.

\section{SIMULATIONS OF TRP-CAGE}

We have performed additional REST2 simulations of the 20-residue Trp-cage (NLYIQWLKDGGPSSGRPPPS) starting from the crystal structure (1L2Y). The REST2 protocol, the number of replicas and the $\lambda$ values were identical to that used for chignolin, while the system composition, the system size and the employed forcefield $\left(\right.$ Amberff99SB $^{10}$ ) were identical to that used in a previous REMD study, ${ }^{11}$ the only difference being that we simulated the protein in its zwitterionic form. The long-range interactions cut-offs and 1-4 interactions were carefully adapted to be compatible with this forcefield in NAMD. The melting curves converge after $\approx 100$ ns and the simulations were propagated up to 250 ns. The fraction of folded states was estimated using a $2.2 \AA$ cutoff for the RMSD to the crystal structure that was suggested before. ${ }^{11}$ For this system, we have used the Amberff99SB forcefield ${ }^{10}$ for two reasons: first, it allows direct comparison with previous REMD simulations performed on the same system; second, 300-ns REST2 simulations with the CHARMM36 forcefield suggested that the protein was only very marginally stable at all temperatures. This is in agreement with previous 0.2 millisecond simulations employing CHARMM22* and showing a serious mismatch between experimental and simulation melting temperatures. ${ }^{9}$

\section{FREE ENERGY PERTURBATION APPROACH FOR MUTATION EFFECTS}

The effect of the double mutations G1Y and G10Y has been estimated by several methods. First we have considered some optimized and fast methodologies available in the market to estimate the free energy change due to single point mutations. In some cases, PoPMusic server ${ }^{12}$ and the Maestro software, ${ }^{13}$ the mutations result destabilising, being associated to a positive $\Delta \Delta G$. The failure of these methods is probably due to a poor account of the solvation contribution, that is rather important in our study case since the mutations are localised at the very solvent exposed peptide terminals. A good estimate, although quantitatively too favourable when compared to experiment, is provided by the Eris webserver. ${ }^{14}$ Using rigid and flexible backbone the $\Delta \Delta G$ is -3.0 and $-4.0 \mathrm{kcal} / \mathrm{mol}$, respectively. The robustness of the Eris approach has been confirmed by checking the reverse mutations and starting from the recently deposited structure of CLN025 (PDB code 2RVD, which was deposited after the completion of the rest of the work). As final supportive check we have performed Free Energy Perturbation (FEP) calculations at $\mathrm{T}=300 \mathrm{~K}$ and using NAMD. Sequential mutations, G1Y and G10Y have been performed starting from the folded state (PDB 1UAO) and from a representative elongate configuration representing the unfolded state as generated by our simulations. The calculations were performed with activated decoupling for hybrid interactions of the mutated aminoacids following recommendation of the NAMD FEP tutorial. FEP estimate of $\Delta \Delta G$ is of $-1.0 \mathrm{kcal} / \mathrm{mol}$, in very good agreement with experiments.
* To whom the correspondence should be addressed. Email: stirnemann@ibpc.fr $\dagger$ To whom the correspondence should be addressed. Email: sterpone@ibpc.fr 
1 Wang, L.; Friesner, R. A.; Berne, B. J. Replica exchange with solute scaling: A more efficient version of replica exchange with solute tempering (REST2). J. Phys. Chem. B 2011, 115, 9431-9438.

${ }^{2}$ Honda, S.; Akiba, T.; Kato, Y. S.; Sawada, Y.; Sekijima, M.; Ishimura, M.; Ooishi, A.; Watanabe, H.; Odahara, T.; Harata, K. Crystal structure of a ten-amino acid protein. J. Am. Chem. Soc. 2008, 130, 15327-15331.

3 Honda, S.; Yamasaki, K.; Sawada, Y.; Morii, H. 10 Residue folded peptide designed by segment statistics. Structure 2004, 12, 1507-1518.

4 Huang, J.; MacKerell, A. D. CHARMM36 all-atom additive protein force field: Validation based on comparison to NMR data. J. Comput. Chem. 2013, 34, 2135-2145.

${ }^{5}$ Phillips, J. C.; Braun, R.; Wang, W.; Gumbart, J.; Tajkhorshid, E.; Villa, E.; Chipot, C.; Skeel, R. D.; Kalé, L.; Schulten, K. Scalable molecular dynamics with NAMD. J. Comput. Chem. 2005, 26, 1781-1802.

${ }^{6}$ Kührová, P.; De Simone, A.; Otyepka, M.; Best, R. B. Force-field dependence of chignolin folding and misfolding: Comparison with experiment and redesign. Biophys. J. 2012, 102, 1897-1906.

7 Huang, K.; García, A. E. Acceleration of lateral equilibration in mixed lipid bilayers using replica exchange with solute tempering. J. Chem. Theo. Comput. 2014, 10, 4264-
4272.

8 Seibert, M. M.; Patriksson, A.; Hess, B.; Van Der Spoel, D. Reproducible polypeptide folding and structure prediction using molecular dynamics simulations. J. Mol. Biol. 2005, $354,173-183$

${ }^{9}$ Lindorff-Larsen, K.; Piana, S.; Dror, R. O.; Shaw, D. E. How fast-folding proteins fold. Science 2011, 334, 517520 .

10 Hornak, V.; Abel, R.; Okur, A.; Strockbine, B.; Roitberg, A.; Simmerling, C. Comparison of multiple Amber force fields and development of improved protein backbone parameters. Proteins 2006, 65, 712-725.

11 Day, R.; Paschek, D.; Garcia, A. E. Microsecond simulations of the folding/ unfolding thermodynamics of the Trp-cage miniprotein. Proteins 2010, 78, 1889-1899.

12 Dehouck, Y.; Grosfils, A.; Folch, B.; Gilis, D.; Bogaerts, P.; Rooman, M. Fast and accurate predictions of protein stability changes upon mutations using statistical potentials and neural networks: PoPMuSiC-2.0. Bioinformatics 2009, 25, 2537-2543.

13 Laimer, J.; Hofer, H.; Fritz, M.; Wegenkittl, S.; Lackner, P. MAESTRO - multi agent stability prediction upon point mutations. BMC Bioinformatics 2015, 16, 116.

14 Yin, S.; Ding, F.; Dokholyan, N. V. Eris: an automated estimator of protein stability. Nature Methods 2007, 4, 466-467. 